\title{
Roberto Cardoso de Oliveira: una Antropología Reflexiva
}

\author{
Gabriel O. Alvarez ${ }^{12}$ \\ 'Programa de Pós-graduação em Antropologia Social, Faculdade de Ciências Sociais, Universidade \\ Federal de Goiás (PPGAS/FCS/UFG), Goiânia/GO, Brasil \\ ${ }^{2}$ Benemérita Universidad Autónoma de Puebla (BUAP), México
}

\section{Resumen}

Este artículo analiza la trayectoria y la obra de Roberto Cardoso de Oliveira. Siguiendo su metodología hermenéutica, se analizan las monografías ejemplares y conceptos claves a lo largo de su trayectoria académica. Esta trayectoria fue dividida en el joven Roberto Cardoso de Oliveira, etnólogo y el viejo Roberto Cardoso de Oliveira, humanista con una mirada hermenéutica. El análisis explora la dimensión reflexiva en la obra del autor, tanto en el trabajo de campo como en la escrita, así como la reflexión que la etnografía despierta en el público. El trabajo finaliza sugiriendo algunos desdoblamientos de la perspectiva antropológica del autor en el campo de la antropología visual, rituales y performances.

Palabras clave: Roberto Cardoso de Oliveira; identidades; antropología interpretativa; hermenéutica. 


\title{
Roberto Cardoso de Oliveira: \\ a Reflexive Anthropology
}

\begin{abstract}
This paper analyses the trajectory and work of Roberto Cardoso de Oliveira. Following his hermeneutical methodology, analysis exemplary monographs and key concepts are analysed throughout his academic career. This trajectory was divided into the young Roberto Cardoso de Oliveira, an ethnologist and the old Roberto Cardoso de Oliveira, a humanist with a hermeneutical perspective. The analysis explores the reflective dimension in the author's work, both in the fieldwork and in the written work, as well as the reflection that ethnography arouses in the public. The work ends by suggesting some insight of the author's anthropological perspective in the field of visual anthropology, rituals and performances.
\end{abstract}

Key words: Roberto Cardoso de Oliveira; identities; interpretative anthropology; hermeneutics.

\section{Roberto Cardoso de Oliveira: uma Antropologia Reflexiva}

\section{Resumo}

Este artigo analisa a trajetória e a obra de Roberto Cardoso de Oliveira. Seguindo sua metodologia hermenêutica, se analisam as monografias exemplares e os conceitos-chave ao longo de sua carreira acadêmica. Essa trajetória foi dividida no jovem Roberto Cardoso de Oliveira, etnólogo, e o velho Roberto Cardoso de Oliveira, humanista com perspectiva hermenêutica. A análise explora a dimensão reflexiva no trabalho do autor, tanto no trabalho de campo quanto no trabalho escrito, bem como a reflexão que a etnografia suscita no público. $O$ trabalho termina sugerindo alguns desdobramentos da perspectiva antropológica do autor no campo da antropologia visual, rituais e performances.

Palavras-chave: Roberto Cardoso de Oliveira; identidades; antropologia interpretativa; hermenêutica. 


\title{
Roberto Cardoso de Oliveira, una Antropología Reflexiva'
}

\author{
Gabriel O. Alvarez
}

Este trabajo presenta los principales puntos del pensamiento antropológico de Roberto Cardoso de Oliveira. Metodológicamente, analizaremos la obra de un autor; prestaremos atención a las monografías ejemplares y los conceptos claves; observaremos quiénes fueron sus interlocutores o, como diría Roberto, su comunidad de comunicación. En este análisis, colocaremos en diálogo la obra del “joven” Roberto Cardoso de Oliveira etnólogo y la visión humanista del "viejo" Roberto Cardoso de Oliveira. Esperamos que esta síntesis permita presentar los principales aportes de Roberto Cardoso de Oliveira para el público de México, que está más familiarizado con el joven teórico de la "fricción interétnica" que con el antropólogo hermeneuta. Como forma de enfatizar la vigencia de las ideas del autor, finalizamos señalando algunos desdoblamientos de esta perspectiva en el análisis de rituales, performance y antropología visual. Esperamos mostrar así la vigencia de su pensamiento a 15 años de su muerte.

\section{El joven Roberto Cardoso de Oliveira: una etnología política}

Roberto Cardoso de Oliveira (1988; Cardoso de Oliveira, Ruben, 1995), en su análisis de las tradiciones en Antropología, siempre prestó especial atención a la institucionalización de la antropología social en la academia. Por este motivo, iniciamos nuestro análisis con la trayectoria institucional de Roberto Cardoso de Oliveira.

Recién graduado en filosofía por la Universidad de São Paulo (USP), el joven Roberto Cardoso de Oliveira fue convidado a asistir a una conferencia de Darcy Ribeiro. Impresionado con la presentación, se acercó para conversar después de la conferencia y recibió la invitación de Darcy Ribeiro, en la época en el Servicio de Protección al Indio (SPI), para incorporarse al órgano indigenista. Durante ese periodo Roberto Cardoso de Oliveira tiene una formación empírica en antropología al tomar contacto con los problemas indígenas, primero a través de los informes, luego a partir de la realización de etnografías. El SPI estaba a cargo de la política indigenista de Brasil y contaba en sus cuadros con tres antropólogos, Darcy Ribeiro (1979), Eduardo Galvão (1979) y el joven Roberto Cardoso de Oliveira, quien participó del órgano entre 1955 y 1958 (Alvarez, 2008).

En 1960, Roberto Cardoso de Oliveira ingresó como profesor en el Museo Nacional en Río de Janeiro, donde tuvo como colega a Luiz de Castro Farias. En el Museo creó primero una especialización en Antropología, que en poco tiempo se transformó en Maestría y en curso de Doctorado. En 1971, realizó un posdoctorado en la Universidad de Harvard, durante su año sabático. En 1972, fue convidado por Roque Laria para crear el Programa de Pos-Graduación en Antropología Social de la Universidad de Brasilia (PPGAS/UnB), institución en la que permaneció hasta 1986. En 1974, viajó a México, donde estuvo vinculado al CIESAS. En este período realizó una etnografía con los Tarascos y estableció fraternos vínculos con los colegas mexicanos, en especial con Bonfil Batalla. Permaneció en la UnB hasta el año 1986, cuando recibió la invitación para vincularse a la UNICAMP,

\footnotetext{
Este artículo fue presentado originalmente como conferencia en el Centro de Investigaciones y Estudios Superiores en Antropología Social (CIESAS) DF/Mx, 14 de mayo de 2019, en el marco de la Cátedra Roberto Cardoso de Oliveira (CIESAS/UNICAMP). Agradezco al colega Mariano Baez Landa (CIESAS) por invitación y a Iván Deance y Verónica Deance, de la BUAP, por la invitación para este periodo como Profesor Visitante en el Laboratorio Universitario Imagen y Memoria, que hicieron posible este periodo en México y la participación en el evento.
} 
con el propósito de crear un programa de Posgrado en Relaciones Sociales, inspirado por la experiencia en Harvard. Permaneció en la UNICAMP por una década. Volvió a la UnB como profesor visitante en el CEPPAC/ UnB, en Brasilia, institución a la que permaneció vinculado hasta su muerte, en 2006.

El propio Roberto Cardoso de Oliveira marcaba una inflexión a lo largo de su vida académica contrastando el joven Roberto, etnógrafo, trabajando con los pueblos indígenas de Brasil y el viejo Roberto, marcado por la preocupación hermenéutica.

El joven Roberto Cardoso de Oliveira realizó sus etnografías con los Terena (1976 [1960]), en el sur de Brasil y con los Tikuna en Amazonas (1996 [1964]). Su visión académica estaba orientada por la antropología social británica y los problemas indicados por la Escuela de Manchester. Trabajó el tema de las identidades indígenas, como construcción ideológica y como posición subordinada en la estructura social. Desarrolló el concepto de "fricción interétnica", donde señaló la contradicción dialéctica entre los pueblos indígenas y la sociedad nacional. En los frentes de expansión, la población nacional se apropia de las tierras indígenas, mas necesita de la mano de obras indígena para la explotación de los territorios. La identidad indígena es estigmatizada, reforzando su posición subordinada en la estructura social. Frente a esta situación, la estrategia de algunos individuos consistía en negar, mimetizar su identidad indígena, asumiendo la identidad de bugre, en el sur, o de caboclo en la región amazónica (ladino, en México). Los que optaron por esta estrategia renunciaron al mundo del indio, pero no por eso fueron incorporados en el mundo del blanco. Subyace a este análisis una crítica a la teoría de la aculturación en boga en la academia americana. Los pueblos indígenas no podían ser analizados aisladamente de sus relaciones con la sociedad nacional.

En esa época, los pueblos originarios no tenían voz, o su voz era la del padre o la de los antropólogos. La relación entre los pueblos indígenas y el estado tenía que ser construida a partir de la formulación de una política indigenista comprometida con el futuro de estos pueblos. Se construía una antropología preocupada por encontrar soluciones para los problemas enfrentados por estas poblaciones que habían sido relegadas por los proyectos nacionales en Latinoamérica, como lo señaló años más tarde la Reunión de Barbados (1970), de la que participaron Bonfil Batalla, Miguel Bartolomé, Darcy Ribeiro, Silvio Coelho, entre otros.

El otro período, que él mismo caracterizaba como el viejo Roberto Cardoso de Oliveira, fue marcado por lo que él llamaba de "guinada" hermenéutica, la guinada puede ser traducida como 'viraje', 'cambio de rumbo', y es donde se manifiesta la preocupación humanista de Roberto Cardoso de Oliveira. Las preocupaciones hermenéuticas, introducidas por Geertz en la antropología americana, fueron repensadas por Roberto Cardoso de Oliveira, con su formación de filósofo, y renovadas con la lectura de autores como Dilthey (1974), Gadamer (1992; 1993), Apel (1985), entre otros. A partir de estas preocupaciones, pasó a ver los paradigmas de la antropología como tradiciones nacionales (Cardoso de Oliveira, 1988). Se destacan en este periodo sus preocupaciones con los diferentes estilos nacionales que adquirió la antropología. Otro tema abordado en este periodo fueron la fusión de horizontes al interior de las comunidades de comunicación, ecuacionadas en términos de moral y ética; y sus reflexiones sobre el trabajo del antropólogo. En este período, a partir de la década de 1980, en Brasil asistimos a la emergencia del movimiento indígena y la escolarización de los pueblos indígenas; un indígena, que a diferencia de lo que ocurría décadas atrás, ahora tiene su propia voz y acceso a los textos escritos por los antropólogos.

Las etnografías escritas por el joven Roberto fueron: Del indio al bugre. El proceso de asimilación entre los Terena, publicada en 1960 (1976 [1960]); El indio y el mundo de los blancos: la situación de los Tikuna en el Alto Solimones, publicada en 1964 (1996 [1964]); Urbanización y tribalismo, publicada en 1968 (1968).

En Del indio al bugre, Roberto Cardoso de Oliveira realiza una etnografía de los Terena que viven en las Tierras Indígenas del grupo en el sur de Brasil (1976 [1960]). Subyace en este trabajo una crítica a la teoría de la aculturación, en boga en la academia americana. Recordemos que la Asociación American a de Antropología (AAA) emitió dos memorandos sobre el tema. Los Terena son un grupo Aruak que no pasado tuvieron una 
rígida estructura social, dividida en tres estratos y en dos mitades. Tradicionalmente, cada uno de estos grupos era endógamo entre sí, una estructura social que no se sustentó con las condiciones que enfrentaron los indígenas después del contacto. En su trabajo de campo, Roberto Cardoso de Oliveira muestra que el grupo mudó su estructura social y las mitades sólo eran invocadas para la realización de rituales, y ya no orientaban los casamientos del grupo. A pesar de estas mudanzas en la estructura social, los Terena mantenían su identidad étnica, que aseguraba su derecho a las tierras en la reserva. El autor también observó el transito urbano de estos indígenas y como algunas costumbres de la ciudad eran practicadas en la tierra indígena, como la vuelta al final del día, paseando por la calle de la aldea.

El indio y el mundo de los blancos es su libro más destacado y uno de los que fue más veces reditado (Cardoso de Oliveira, 1996 [1964]). En su etnografía sobre los Tikuna, acuña el concepto clave de fricción interétnica que orientará la antropología del joven Roberto Cardoso de Oliveira. En este trabajo, analiza la situación de este grupo indígena localizado en el Alto Solimones, en la zona de explotación del caucho. El autor observa la contradicción dialéctica entre el frente de expansión de la sociedad nacional y las poblaciones nativas. Los blancos necesitaban del territorio indígena y de la mano de obra indígena para la explotación del caucho. La estrategia de los blancos fue someter a los indígenas a través de la esclavitud por deudas. Si la sociedad nacional está estructurada en clases sociales, subyace un corte étnico, que coloca a los indígenas en la posición más baja en la estructura social. En el libro indica el doble carácter de las identidades, como construcción ideológica y como posición en la estructura social. La posición subordinada en la estructura social era reforzada por una serie de estereotipos que estigmatizaban la imagen del indio. Frente a esta situación, una de las estrategias adoptadas por los indígenas consistía en negar su identidad indígena para asumirse como "caboclos", que podría ser traducido como 'ladinos' en México. El caboclo niega su identidad indígena como forma de mimetizarse para escapar del estigma que sufren los indios. El autor señala que los que optaron por esta estrategia quedaron en el peor de los mundos, porque salieron del mundo del indio sin ser incorporados en el mundo del blanco.

Urbanización y tribalismo (Cardoso de Oliveira, 1968) fue la primera etnografía sobre indígenas urbanos realizada en Brasil. En este trabajo, contó con la participación de alumnos del programa de especialización en Antropología Social que había iniciado en el Museo Nacional. Esta investigación se centró en los indígenas Terena que habían migrado a las ciudades próximas de las tierras indígenas. En su etnografía, reconstruye las redes de relaciones sociales movilizadas en esas migraciones, para mostrar que las estrategias envolvían elementos tradicionales como el parentesco. Analizó incluso los agrupamientos urbanos como Aldeinha que congregaban varias familias extensas y contaba con la presencia de un chamán que atendía a los Terena y al público local. En este trabajo, describió la inserción de los indígenas en el mercado de trabajo urbano, en posiciones subordinadas, como peones en la construcción, en panaderías e incluso como portero de un cine. En sus entrevistas los indígenas revelan que el trabajo urbano era menos pesado que el trabajo campesino en la tierra indígena. Los indígenas se reorganizaron en el medio urbano, cambiaron su estructura social, mas mantuvieron su identidad indígena. La aculturación no es un fenómeno que afecte al grupo como un todo. Algunos individuos pueden negar su identidad indígena y presentarse como bugres, mas buena parte de la población Terena continúa invocando su identidad indígena inclusive en el medio urbano. Urbanización y tribalismo reveló la implementación de mecanismos tradicionales en el medio urbano y el tránsito, entre las aldeas y la ciudad.

Sus principales interlocutores en la época eran sus colegas del SPI, Darcy Ribeiro y Eduardo Galvão. Darcy, había realizado etnografías con los Kadiweu y con los Kayapó, su punto de vista teórico estaba inspirado en el evolucionismo multilinear y había focalizado su atención en los frentes de contacto de la sociedad nacional con los pueblos indígenas (Ribeiro, 1979). En sus libros apuntaba los efectos devastadores del contacto, tanto por las matanzas provocadas por los blancos para apropiarse de las tierras indígenas, como por la mortandad de indígenas provocada por el contagio de enfermedades para las cuales las poblaciones nativas no tenían defensas. 
Eduardo Galvão (1979) realizó su etnografía con los Tenethara, bajo la orientación de Charles Wagley, antropólogo americano. La influencia de Wagley le dio una orientación funcionalista al trabajo. Otro interlocutor importante fue su orientador de Doctorado, el profesor Florestan Fernandes, destacado sociólogo de la USP. Sociólogo de formación, Florestan publicó dos libros sobre los Tupi-Guaraní en los que reconstruye la organización social del grupo, a partir de los documentos de los cronistas de la conquista. Otro de los temas de interés de Florestan Fernandes fue la inserción del negro en la sociedad nacional después de la abolición de la esclavitud. Uno de sus libros más importantes sobre el tema, El negro y el mundo del blanco (Fernandes, 1972), parafrasea el nombre del libro de Roberto sobre los Tikuna. Un raro homenaje del orientador al orientando. Otros interlocutores de la época fueron Fernando Henrique Cardoso y Octavio Ianni, con quienes se reunían en un seminario para discutir la obra de Marx, en un departamento en São Paulo, fuera de la Universidad. La mirada teórica del joven Roberto Cardoso de Oliveira estaba orientada por la Antropología Social Británica y, en especial, la Escuela de Manchester, que tenía un diálogo con el marxismo.

En la producción del joven Roberto Cardoso de Oliveira sigue una serie de libros con compilación de artículos que son reflexiones que se desprenden de sus etnografías, o que se originaron en ellas. Me refiero a La sociología del Brasil indígena (1978 [1972]); a Enigmas y soluciones (1983); y a su trabajo clásico, Identidad, etnia y estructura social (1978). Los dos primeros son una compilación de artículos, muchos de los cuales presentan los resultados del proyecto Harvard-Brasil Central, que desenvolvió en el Museo Nacional, con Maybury-Lewis como contraparte por la universidad americana. En estos trabajos se explora la aplicación del concepto de fricción interétnica en las diversas regiones de Brasil, analizando también los diferentes frentes de expansión de la sociedad nacional. Los trabajos presentan los problemas enfrentados por los pueblos originarios en una relación de colonialismo interno. Uno de ellos analiza el movimiento mesiánico de la Santa Cruz entre los Tikuna, así como la influencia de la circulación del dinero entre estos grupos y las migraciones provocadas por el movimiento mesiánico. Estos libros traen al lector tanto la situación enfrentada por las poblaciones indígenas, cuanto la búsqueda por soluciones para estos problemas. Una solución que pasa necesariamente por la implantación de políticas públicas de los estados que contemplen a las poblaciones nativas. Enigmas y soluciones (1983) compila los artículos de carácter más etnográfico y La sociología del Brasil indígena (1978 [1972]) los trabajos centrados en la política indigenista y el colonialismo interno del estado brasilero.

El proyecto Harvard-Brasil Central/Fricción Interétnica fue importante para la consolidación del programa de Posgrado en Antropología Social en el Museo Nacional de Río de Janeiro. Este proyecto permitió el intercambio de docentes y estudiantes, así como estimuló la realización de etnografías sobre los pueblos indígenas. Pasaron por el proyecto sus alumnos del curso de especialización: Roberto da Matta, Roque Laraia, Julio Cezar Melatti.

Identidad, etnia y estructura social (1978), su trabajo ejemplar de la época, fue escrito durante su período de posdoctorado en Harvard y se complementa con un capítulo escrito durante su estadía en México en 1974. En las bibliotecas de Harvard, Roberto Cardoso de Oliveira descubre los trabajos de Fredrick Barth (2000), formado en la escuela de Manchester, que vienen al encuentro de las reflexiones desarrolladas en sus etnografías. En primer lugar, quebrar la falsa ecuación, de sentido común, de que una identidad es igual a una cultura, a una sociedad, a una lengua, a una religión. Quebrada esta falsa ecuación, Barth proponía ver las identidades como formas de organización social; las identidades se organizan en sistemas interétnicos; la importancia de los límites de las identidades. Límites éstos que pueden ser atravesados por individuos, pero no por grupos enteros, como suponía el concepto de aculturación. Roberto Cardoso de Oliveira propone ver las identidades como brújulas que orientan las redes de relaciones sociales. Desarrolla teóricamente la idea del doble carácter de las identidades, como construcción ideológica y como posición en la estructura social. Si las identidades tienen una gramática, en su articulación en sistemas interétnicos, en América Latina, en el caso de las poblaciones indígenas, la misma aparece condicionada por el colonialismo interno desplegado por los estados nacionales y sus frentes de expansión. 
El concepto de "colonialismo interno" había sido formulado por Pablo González Casanova en México e incorporado por Roberto Cardoso de Oliveira en su concepto de fricción interétnica. Durante este período, Roberto participa de un intercambio con México, donde florecía, en la época, la antropología aplicada y se consolidaba el CIESAS como un ambicioso programa antropológico. En sus viajes a México forjó una entrañable amistad con Bonfil Batalla, su gran compañero en el CIESAS D.F., así como con otros colegas como Teresa Rojas, Arturo Warman y, en el CIESAS de Oaxaca, con Salomón Nahmad Sitton. Durante su estancia en México realizó una experiencia etnográfica con los Tarasco y escribió un nuevo capítulo, incluido en la segunda edición de Identidad etnía y estructura social. En esa época, su libro Urbanización y Tribalismo fue publicado en México, en 1972, por el Instituto Indigenista Interamericano.

Eran los años de la Declaración de Barbados (1970), que denunciaba la situación enfrentada por las poblaciones indígenas de las tierras bajas sudamericanas, que resultaba en el genocidio y etnocidio de estas poblaciones. La Declaración apuntaba la responsabilidad de los estados, por acción y por omisión frente a la situación, producto de negligencia política y delegación de la tutela de estas poblaciones en manos de la Iglesia Católica y las sectas protestantes. Participaron de esta reunión Miguel Bartolomé, Darcy Ribeiro, Silvio Coelho dos Santos, Guillermo Bonfil Batalla entre otros. A pesar de no haber participado del encuentro, las reflexiones de Roberto Cardoso de Oliveira estaban presentes en los debates. Roberto Cardoso de Oliveira participó años más tarde en el encuentro de Costa Rica, una reunión de la UNESCO donde se discutió, junto con Stefano Varese y de líderes indígenas, el concepto de etnodesarrollo, en 1981.

\section{La "guinada" hermenéutica}

A partir de la década de 1980, inspirado inicialmente en los trabajos de Geertz, que reintroducen la hermenéutica en la antropología americana, Roberto Cardoso de Oliveira da una "guinada"-como gustaba decir- un cambio de rumbo, dando lugar a un Roberto Cardoso de Oliveira humanista. Si bien en esta nueva fase dialoga con Geertz, su abordaje hermenéutico es más profundo. Mientras el autor americano se apoya principalmente en Paul Ricoeur, Roberto Cardoso de Oliveira reflexiona a partir de su formación inicial como filósofo, para incorporar autores como Dilthey (1974), Gadamer (1992; 1993) y Apel (1985). Sus reflexiones se vuelven hacia la antropología como Weltanshauung, como tradición, visión de mundo.

Esta mudanza de preocupaciones se refleja en sus libros Compilación e introducción a la obra de Marcel Mauss (1979) y W. H. R. Rivers (1985); Sobre el pensamiento antropológico (1988); La Antropología de Rivers (1991); Razón y afectividad: el pensamiento de L. Lévy-Bruhl (1991); y Estilos de Antropología (1995).

Las dos primeras obras del grupo de trabajos son compilaciones de artículos de Mauss y de Rivers. El método que está por detrás de estas compilaciones es analizar la obra del autor y seleccionar sus trabajos ejemplares; contextualizar la obra, al interior de una tradición académica, con sus antecedentes, sus interlocutores y sus desdoblamientos. Este método se aplica también a sus estudios monográficos sobre Rivers (Cardoso de Oliveira, 1985, 1991) y Lévy-Bruhl (Cardoso de Oliveira, 2002 [1991]). Marcel Mauss y Rivers tuvieron un papel central en la institucionalización de las tradiciones francesa y británica de antropología social.

$\mathrm{Al}$ analizar la obra de Rivers, da merecido destaque al método genealógico, que permite visualizar las redes de relaciones sociales, algo que el ojo no percibe. El autor señala la importancia del método genealógico como análogo a la importancia del microscopio para el biólogo. El otro punto destacado es el fieldwork, el trabajo de campo, empírico, aplicado a las investigaciones en la expedición al Estrecho de Torres, de la cual participaron Haddon, Seligman y Rivers, y que le dieron una saludable impronta empírica a la antropología social británica.

$\mathrm{Al}$ analizar la obra de Marcel Mauss, destaca el papel que ocupó el estudio de las categorías del espíritu humano en la tradición francesa. El concepto de categorías fue discutido por Aristóteles. Kant, con la idea de la razón trascendental, retoma el tema de las categorías del espíritu humano, pero coloca el tiempo y el espacio 
como datos de lo sensible. Esta tesis es contestada por Durkheim (1989) en las Formas elementares de la vida religiosa [1912]. Durkheim analiza el material sobre las tribus australianas para mostrar que tiempo y espacio, para ser construidos, deben estar marcados por algún quiebre que permita su conceptualización. Encuentra esta diferenciación en la atribución de los valores de sagrado y profano, tanto al espacio de la aldea, como al tiempo de fiesta y el tiempo profano del trabajo cotidiano. A partir de la atribución de estos valores es que la sociedad se percibe como persona moral, como colectivo y se constituyen las categorías de totalidad, tiempo y espacio.

Estas ideas fueron retomadas por Marcel Mauss (Cardoso de Oliveira, 2003 [1950]) y por otros participantes de la escuela francesa de Antropología. Las categorías, son comunes a toda la humanidad, pero diferentes en cada sociedad. Está idea fue explorada por Mauss en "La noción de persona", "Las técnicas del cuerpo", "Ensayo sobre el don", la muerte, sistemas clasificatorios. En el "Ensayo sobre el don" realizó una crítica certera a los teóricos del contractualismo, al señalar que no podemos colocar la forma contrato (mercantilista) como origen de la sociedad. Por el contrario, los mecanismos del don -la obligación de dar, obligación de recibir y obligación de retribuir- crean relaciones sociales, son más elementares y universales que la forma contrato, surgida durante el mercantilismo. Otra preocupación que aparece en Mauss es con estas categorías como formas tradicionales y eficaces. Mauss aplicó esta fórmula, tanto para los rituales y oraciones, como para las técnicas del cuerpo. Colocó las categorías al interior de tradiciones culturales, y las mismas tienen que ser evaluadas en términos de eficacia. El tema de las categorías también fue trabajado por otros autores. Herzfield trabajo la oposición entre mano derecha y mano izquierda como categoría, y más recientemente Dumont, al diferenciar los sistemas de parentesco consanguíneos de los sistemas dradivianos, donde las relaciones de afinidad preceden a la unión matrimonial.

Cardoso de Oliveira (2002 [1991]), al analizar la obra de Levy Bruhl en términos de las categorías de la escuela francesa, muestra que el autor nos coloca frente a dos categorías, aquella del pensamiento lógico, basado en el principio de la contradicción, y otra en que el pensamiento no se rige por el principio de la contradicción del racionalismo y puede estar regido por el principio de la transformación, como el caso del pensamiento amerindio. En última instancia, lo que orienta el pensamiento no es la lógica, sino los valores. En este punto, el autor elaboró el principio de participación para explorar cómo los valores movilizan a partir de las emociones, de los sentimientos.

El trabajo ejemplar de este período es Sobre el pensamiento antropológico (Cardoso de Oliveira, 1988), donde el autor completa su "guinada" hermenéutica y nos presenta una visión plural de la antropología. Para entender la dimensión de esta "guinada", debemos comparar la idea de ciencia desde la perspectiva positivista y colocar, como contrapunto, el punto de vista hermenéutico. Para el positivismo, el conocimiento científico es producto de la aplicación del método. El conocimiento científico buscaría la explicación por medio de leyes, a partir del método experimental. Estas leyes tendrían el poder de predecir y de articularse en un sistema de leyes universales, ahistóricas y objetivas. Estas ideas positivistas acerca de la ciencia ya fueron cuestionada por Thomas Kuhn (1975) en la revolución científica. Este autor señaló que los avances en la ciencia no se dan por la comprobación o refutación de las hipótesis, sino por cambios en los paradigmas científicos. La ciencia no es una entidad autónoma, se sustenta en una comunidad científica y la mudanza de paradigma se relaciona con la creación de un nuevo consenso en torno de las cuestiones relevantes. La ciencia depende de una comunidad de investigadores, y la misma puede ser pensada como una Comunidad de Comunicación (Apel, 1985).

En su abordaje humanista, Roberto Cardoso de Oliveira (1988) plantea, desde un punto de vista hermenéutico, la importancia de la comprensión, por sobre la explicación. En su crítica va más allá de Kuhn, al afirmar que, en el caso de las ciencias sociales, esas comunidades científicas estaban orientadas nacionalmente. Así, mientras que en la física los diferentes paradigmas se suceden en el tiempo, en la antropología coexisten diversos paradigmas, producto de tradiciones nacionales. Al ver a la ciencia como Weltanshauung, el conocimiento deja 
de pretenderse universal y adquiere el carácter de tradiciones nacionales; es un conocimiento contextualizado históricamente; no se trata de un conocimiento objetivo, es un conocimiento intersubjetivo, producto del consenso al interior de una comunidad de investigadores.

Podemos ver así el desenvolvimiento de diferentes tradiciones nacionales en el campo de la antropología, lo que nos lleva a comprender la coexistencia de diferentes paradigmas, anclados en estas Weltanshauung, visiones de mundo. Para visualizar estas tradiciones, Roberto Cardoso de Oliveira (1988) usaba como recurso la creación de una matriz disciplinar, en las que colocaba: el paradigma histórico cultural de la antropología boasiana; la tradición francesa, con su preocupación en las categorías; la tradición británica con su empirismo; finalmente, la nueva tradición interpretativa, inaugurada por Geertz. La matriz nos permite colocar en su interior los principales autores claves y las monografías ejemplares de cada una de estas tradiciones. Por otro lado, nos permite explorar las áreas de tensión entre los diferentes paradigmas. Un dato que puede pasar desapercibido para el lector es la importancia que daba Roberto Cardoso de Oliveira al momento de la institucionalización de la disciplina en la Universidad, una vez que los autores analizados tuvieron un papel de destaque en la creación de los cursos de antropología en sus respectivos países.

Las tradiciones presentadas en la matriz son las de los países centrales. Roberto Cardoso de Oliveira (1988; Cardoso de Oliveira, Ruben, 1995) prestó también atención al desarrollo de la antropología en los países periféricos. La relación entre la antropología de los países centrales y periféricos no debe ser leída en clave de la teoría de la dependencia. Por el contrario, lo que apuntaba el profesor es que mientras que los antropólogos de los países centrales son socializados en el seno de un paradigma, nosotros, formados en las antropologías periféricas, podemos transitar por los diferentes paradigmas, explorar sus áreas de tensión, desenvolver una visión plural de la antropología.

El análisis de las antropologías periféricas tiene su continuidad en el libro Estilos de antropología (1995), coordinado junto con Guilherme Ruben, donde presentan los resultados del estudio comparativo de diferentes "antropologías nacionales" desarrollado por diferentes participantes del proyecto. Roberto trabajó el desarrollo de la antropología española, con énfasis en la antropología catalana, en Barcelona, lo que permitió también explorar las tensiones nacionales al interior del reino de España.

Tenemos otro conjunto de libros, producidos en sus últimos años de vida, en los que explora la cuestión hermenéutica a partir de la antropologización de las cuestiones expuestas por los filósofos. Me refiero a Ensayos antropológicos sobre moral y ética (1996); El trabajo del antropólogo (1998); Los diarios y sus márgenes: viaje a los territorios Terena y Tikuna (2002); y Caminos de la identidad (2006).

Un trabajo clave de este periodo es el artículo "El oficio de antropólogo" que da nombre el libro homónimo. En este trabajo, Roberto Cardoso de Oliveira revisita las preocupaciones de Geertz (1989) sobre la antropología, el estar en el campo y en la academia, ahora desdoblada a partir de las preocupaciones hermenéuticas de Roberto Cardoso de Oliveira y sus experiencias de trabajo de campo (1998).

El autor nos llama la atención sobre tres características de nuestro oficio de antropólogos: el mirar, escuchar y escribir. El mirar y escuchar no deben ser entendidos como datos de lo sensible (como Kant entendía el tiempo y el espacio). La mirada a la que se refiere Roberto Cardos de Oliveira (1998) es una mirada informada, informada por el paradigma, las cuestiones teóricas y el estilo antropológico del investigador. El escuchar se refiere al proceso de fusión de horizontes de comunicación que acontece durante el trabajo de campo. El mismo no se reduce a la práctica de entrevistas, sino que envuelve complejas formas de comunicación, como rituales y performances; diversas formas de conocimiento, como la empatía; y los momentos metódicos y no-metódicos del trabajo de campo. La tercera característica, el escribir, trasciende el mero ejercicio de pasar el pensamiento para el plano escrito e incluye el debate entre pares en la academia. 
A partir de la realización de estas actividades propias de nuestro métier de antropólogos, Roberto Cardos de Oliveira señala los diversos momentos interpretativos de la antropología. En su diálogo con Geertz (1989), Roberto Cardoso de Oliveira los apunta como momentos interpretativos, más fiel a sus enseñanzas en el aula, me gustaría señalar que los mismos son, en realidad, momentos reflexivos.

El primer momento interpretativo-reflexivo acontece durante el trabajo de campo, envuelve el mirar y escuchar, como indicamos anteriormente, una mirada informada teóricamente, marcada por un estilo de hacer antropología y el escuchar como proceso de fusión de horizontes de comunicación. El concepto de fusión de horizontes de comunicación, tomado de Gadamer (1992; 1993), tiene que ver con la comprensión de los horizontes de comunicación del otro, en un proceso que envuelve momentos metódicos y no-metódicos. Durante el trabajo de campo experimentamos un extrañamiento de nuestra propia cultura para poder comprender, a partir de la experiencia, el punto de vista del otro. Este proceso de fusión de horizontes puede envolver recursos metódicos desenvueltos por la disciplina, como entrevistas y genealogías, pero también envuelve otras formas de conocimiento no metódico como el arte, la religión, la música, las comidas, la empatía. A partir de estas consideraciones queda claro que la etnografía no es descripción, es reflexión sobre un problema teórico a partir de la experiencia.

El segundo momento interpretativo-reflexivo es el que se realiza en la academia, cuando traducimos la experiencia del trabajo de campo para un público más amplio. En la academia, cuando presentamos y discutimos los resultados de la investigación, tiene lugar un momento reflexivo entre pares. Las presentaciones en congresos y en las clases, implican también un tipo de reflexión plural, al incorporar las reacciones del público y cuestionamientos a nuestras reflexiones sobre el trabajo de campo. Los datos del trabajo de campo son contrastados con las discusiones levantadas por los colegas en la bibliografía sistematizada. Cuando escribimos, no sólo volcamos nuestras reflexiones en el papel, también volvemos al campo, ahora mediante la evocación, cuando al mirar una de las páginas del cuaderno de campo recordamos las situaciones vividas en el campo, muchas de ellas no registradas en nuestras bitácoras. El producto de este segundo momento reflexivo es una narrativa, en forma de libro, artículo e inclusive video etnográfico. Quiero llamar la atención aquí para una cuestión de estilo de la antropología: las monografías envuelven una forma dramática, son presentadas como un drama.

El tercer momento interpretativo acontece cuando el público lector entra en contacto con la obra y en ese momento el autor no está al lado para comentar lo que quiso decir. Lo que marca este momento son las reflexiones que la obra pueda despertar en la mente del lector. Como drama, la narrativa cautiva al lector y le permite interpretar la experiencia que vivimos durante el trabajo de campo, nuestras reflexiones. En la forma dramática, el significado es producto de la totalidad, resultado del drama. La interpretación del drama envuelve también momentos catárticos que llevan a que el público reflexione a partir de la identificación con los personajes.

Un trabajo revelador de la importancia de la dimensión reflexiva de Roberto Cardoso de Oliveira es El diario y sus márgenes. En este libro, el autor publica los diarios escritos por el joven RCO en sus trabajos de campo con los Terena y los Tikuna, con las márgenes comentadas por el viejo RCO, que vuelve al campo mediante la evocación provocada por los diarios. El drama, coloca en diálogo el joven RCO etnógrafo, empírico, con el RCO humanista, con sus reflexiones de inspiración hermenéutica. Este libro fue pensado para un público amplio, que incluye los indígenas, descendientes de los que aparecen retratados en los diarios. El mismo fue pensado como una restitución, para que los indígenas de hoy, alfabetizados y con voz propia, puedan acceder a los registros de la situación de sus ancestrales hace 50 años atrás, cuando el indio no tenía voz (Alvarez, 2008).

El otro grupo de trabajos se encuentra disperso en los libros Ensayos antropológicos sobre moral y ética (1996); El oficio del antropólogo (1998); Caminos de la identidad. Ensayos sobre etnicidad y multiculturalismo (2006). En los mismos hay un intento del autor para domesticar, en términos antropológicos, 
conceptos formulados por la filosofía. Entre los mismos se destacan: Comunidades de Comunicación, de Apel (1985); la lógica de la acción comunicativa, de Habermas (1989); y la hermenéutica de Gadamer (1992; 1993) y Dilthey (1974).

El concepto de Comunidad de Comunicación de Apel (1985) supone una Comunidad de Comunicación Ideal (CCI) que, por contraste, permite analizar la Comunidad de Comunicación Real (CCR), que podemos relevar durante el trabajo de campo o mediante la aplicación a casos ejemplares, con fines analíticos. Roberto Cardoso de Oliveira enfatizaba en la importancia de la CCI por su carácter revelador, en el sentido fotográfico, cuando se la aplica para analizar las diferencias con la CCR. Es importante destacar las condiciones de la Comunidad de Comunicación Ideal: que la comunicación sea en el plano del discurso; que haya igualdad de condiciones de poder entre las partes; que exista un consenso en torno de las reglas que rigen la comunidad de comunicación; el establecimiento de relaciones dialógicas que deben acontecer en un plano simétrico, en el que tenga lugar una interlocución democrática, entre actores soberanos. La creación de una comunidad de comunicación implica la emergencia de una ética, de un consenso en torno de reglas, límites y valores que hacen el diálogo posible.

Por otro lado, en toda comunidad de comunicación existe una comunidad de argumentación, que constituye su núcleo duro. La lógica de la acción comunicativa enfatiza la importancia del conjunto de reglas que rigen la comunidad de comunicación. Este conjunto de reglas es el que nos coloca en el plano de la ética de acción comunicativa. Notemos que Habermas (1989) trabaja la ética en el plano del discurso. Este autor también trabajó en diversos libros el tema de la esfera pública, como forma de auto organización de la sociedad (1984).

En sus escritos de moral y ética, Cardoso de Oliveira $(1996 ; 1998$; 2006) coloca la moral como el "buen vivir", como tradición, cultura, Weltanshauung. La ética aparece como ética del discurso, como sistema de reglas para la Comunidad de Comunicación interétnica. Cómo analizar el caso de comunidades de comunicación que envuelven diferentes morales, diferentes tradiciones. Aplica estas cuestiones a diversos casos, como el ejemplo de monjas que, en diálogo, en el plano de la argumentación, convencieron a los Tapirapé de abandonar práctica de infanticidio. Otro ejemplo es en la problematización de lógicas de la acción comunicativa y las diferentes esferas: la microesfera, local, que podemos registrar en el trabajo de campo; la mesoesfera nacional, para la cual son invocadas razones de estado; y la macroesfera global, que se materializa en las organizaciones multilaterales. Son numerosos los casos en que los actores de la microesfera, como poblaciones indígenas, consiguieron el apoyo a nivel de organismos multilateral en sus reclamos frente al Estado. Un punto a destacar es que la creación de comunidades de comunicación implica intersubjetividad, una fusión de horizontes de comunicación.

Caminos de la identidad (2006), su último libro, revisita reflexivamente los trabajos sobre identidad del joven Roberto Cardoso de Oliveira. En esos escritos, la identidad había sido separada analíticamente de la cultura, enfatizando sus aspectos de organización social en sistemas interétnicos que estigmatizaban las poblaciones indígenas. El Roberto Cardoso de Oliveira hermeneuta reconoce que las identidades étnicas tienen una espesura en términos de tradiciones culturales, que puede y debe ser trabajada empíricamente. Los estudios de la década de 1960 indicaban la estigmatización de las identidades indígenas y la manipulación de identidades, su negación. La organización del movimiento indígena a partir de la década de 1980 mostró que las luchas por el reconocimiento refuerzan la autoestima de los pueblos. El movimiento que se inició con la lucha por los derechos territoriales continuó en otras áreas, como acceso a las políticas de salud, educación. La lucha por el reconocimiento anclado en las identidades favoreció la autoestima de los grupos y permitió avances en términos de ciudadanía. Las identidades son invocadas en estas reivindicaciones políticas. Roberto Cardoso de Oliveira apunta también los procesos de politización de las identidades en Brasil, México y España, donde realizó trabajos de campo. En este nuevo contexto, el papel del antropólogo es traducir las demandas de esas microesferas locales donde realizamos el trabajo de campo para producir reflexiones en otras arenas, la mesoesfera nacional y la macroesfera global. 
En síntesis, analizamos la producción bibliográfica de Roberto Cardoso de Oliveira en dos bloques, el joven RCO y el RCO humanista, hermeneuta. Cuando revisamos la bibliografía del joven RCO, contrastamos las etnografías Terena y Tikuna, con sus reflexiones sobre la situación indígena, con una serie de libros que lo suceden y son desdoblamientos reflexivos, sea teóricamente, sea como resultado de proyectos inspirados en esa experiencia etnográfica. El joven Roberto Cardoso de Oliveira, como teórico, reflexionó sobre los datos empíricos de su etnografía. Cuando revisamos la obra de Roberto Cardoso de Oliveira como hermeneuta, observamos que sus reflexiones se vuelven sobre la antropología como tradición, como visión de mundo y sus reflexiones sobre la práctica de la etnografía. Nos preocupamos con esta dimensión reflexiva, porque es central en el pasaje de la explicación para la comprensión, la Verstehen de los hermeneutas. En sus consideraciones sobre el oficio del antropólogo, Roberto Cardoso de Oliveira (1998) deja ver la reflexión/interpretación: durante el trabajo de campo, en el encuentro cara a cara con el otro en la experiencia; la reflexión entre pares en la academia; y la reflexión en el público lector, tanto en la reflexión sobre el significado de un drama (la etnografía como drama), como en la reflexión al interpretar un drama a la luz de otros dramas. Esta dimensión reflexiva hermenéutica, Verstehen, aparece también al interpretar al interior de una tradición, y en la reflexión mediante la evocación, en el diálogo que él mismo establece entre el joven Roberto Cardoso de Oliveira etnólogo y el Roberto Cardoso de Oliveira humanista, con sus preocupaciones hermenéuticas.

\section{Desdoblamientos}

Durante mi trabajo de campo en México, un mariachi me dijo: "Los muertos solo mueren cuando nos olvidamos de ellos". Creo que una forma de mantener vivas las enseñanzas de Roberto Cardoso de Oliveira - que falleció en 2006 - es reflexionar sobre cómo nos apropiamos de ellas.

Cuando frecuenté los cursos del profesor Roberto Cardoso de Oliveira intenté la antropologización de los conceptos de Apel $(1985)$ y Habermas $(1984,1989)$ en torno de las comunidades de comunicación para la construcción de mi objeto en la tesis de doctorado. Trabajé con la hipótesis del Mercosur como espacio público (habermasiano) a ser explorado con una etnografía de la comunidad de argumentación que se creó entre políticos y diplomáticos que trabajaban en la implementación de la propuesta. El resultado fue la etnografía: Mercosur Ritual (Alvarez, 200o).

"Ser diplomático es decir una cosa, pensar otra y hacer otra diferente", me dijo un informante durante el trabajo de campo. En efecto, las declaraciones de los participantes eran discordantes y la información de las entrevistas no estaba en armonía con los datos de la economía política, ni con las declaraciones oficiales que balizan el proceso. Fue allí, en el ritual, donde encontré las claves para ordenar la etnografía. El ritual como poderoso aparato comunicacional que se sobreponía a la comunicación racional. Organicé la etnografía como una serie de rituales, de los más laxos, como fiestas de embajadas, a los más rígidos, como reuniones presidenciales. Había focalizado en los diplomáticos y los políticos, pero el trabajo de campo nos reveló un tercer actor, los periodistas, claves para el pasaje de la Comunidad de Comunicación a la Comunidad Imaginada. La comunicación no se da sólo en el plano del discurso. Con frecuencia los rituales se sobreponen a los discursos y los bañan de sentido.

En la investigación Tradición y política Sateré-Mawé(Alvarez, 2009) incorporé en el proyecto la consideración del Roberto Cardoso de Oliveira humanista de que las identidades tienen una espesura, una tradición, visión de mundo que tiene que ser comprendida. En esta oportunidad, el ritual fue la puerta de acceso a esa alteridad radical. En esta investigación incluí el video como herramienta metodológica en el trabajo de campo. Como técnica para alcanzar una antropología compartida, utilicé la cámara participante y la edición compartida. Después de los registros, mostraba el material al grupo; en cada viaje llevaba los clips editados, los presentaba para el grupo y prestaba atención en sus reacciones y finalmente edité el material con la colaboración de un 
joven profesor indígena. Lo más interesante del proceso fue cuando, después de ver el material, el cacique general dijo que quería agregar unas palabras. Las mismas no sólo fueron pertinentes, sino que también indicaron cosas a las que no había prestado la debida atención. El video permite que el grupo reflexiones y participe en los rumbos de la investigación, creando una dimensión dialógica que difícilmente conseguiríamos con el texto escrito.

Para finalizar, algunas consideraciones sobre la etnografía como momento reflexivo. La etnografía no es descripción de los datos de lo sensible, lo que veo y lo que escucho a partir de lo cual voy a escribir una descripción. La "mirada" es una mirada informada por el problema teórico y por el "estilo" de la tradición antropológica. "Escuchar" es un proceso de fusión de horizontes de comunicación que no se restringe al plano del discurso. Conocer a partir del discurso es diferente de conocer a partir de la experiencia. Los rituales y performance también deben ser vistos como sofisticadas formas de comunicación, que tienen menor margen de manipulación que los discursos. La comunicación no es discursiva, es performática, inclusive el discurso incluye una performance al ser actuado frente al interlocutor. Esto nos lleva a apuntar el contraste entre la Comunidad de Comunicación Ideal, donde prevalece el plano del discurso, y la Comunidad de Comunicación Performática, que vivenciamos en los trabajos de campo.

Cuando llevamos las metáforas de "mirar" y "escuchar" hacia el campo de la antropología visual, el video no está en el "mirar". Si pensamos el "escuchar" como proceso de fusión de horizontes de comunicación, con el video o la fotografía estamos escuchando al grupo, no sólo por medio de testimonios, sino principalmente, por medio de performances. La metodología participativa en el uso del video implica también un horizonte ético de alcanzar una antropología compartida, en la que el grupo tenga voz en la construcción del material que lo representará. El uso del video permite también generar un momento reflexivo en el grupo, que tiene importancia para los rumbos de la investigación. Anteriormente destacamos los momentos metódicos y no-metódicos de la investigación y las diversas formas de conocimiento. El video nos permite incorporar aspectos estéticos, arte, música, sensorialidad. A través de la antropología visual podemos mostrar cosas que no se ven y escuchar cosas que no son dichas, performances que no se reducen a datos de lo sensible y son producto de complejas experiencias de campo, con sus momentos reflexivos (Alvarez, 2017).

Sumisión: 19/03/2020

Aprobación: 28/05/2020

Revisión: Federico Lavezzo 


\section{Referencias bibliográficas}

ALVAREZ, Gabriel O. 2000. MERCOSUR Ritual, Políticos y diplomáticos en la política de integración del Cono Sul. Tese de Doutorado em Antropologia. Brasília: Programa de Pós-graduação em Antropologia Social, Departamento de Antropologia, Universidade de Brasília.

ALVAREZ, Gabriel O. 2008. Os Terena e outros temas. A antropologia de Roberto Cardoso de Oliveira. (Vídeo). Brasilia: ABA. https:||youtu.be/Z_dCvBpRmdc

ALVAREZ, Gabriel O. 2009. Satereria. Tradição e política Sateré-Mawé. Manaus: Valer.

ALVAREZ, Gabriel O. 2017. "Antropología visual, performance y hermenéutica: experiencia de ver, escuchar y participar en Huautla de Jimenez (Oaxaca, México)”. In: Mariano Báez Landa \& Gabriel O. Álvarez (orgs.), Um olhar in(com)formado: teorias e práticas na antropologia visual. Goiânia: Editora da Imprensa Universitária. pp. 145-194.

APEL, Karl-Otto. 1985. La transformación de la filosofía. Tomo II. Madrid: Tauros.

BARTH, Fredrik. 200o. "Os grupos étnicos e suas fronteiras". In: LASK, Tomke (org.) O guru, o iniciador e outras variações antropológicas. Rio de Janeiro: Contracapa.

CARDOSO de OLIVEIRA, Roberto. 1976 [1960]. Do Índio ao Bugre. Rio de Janeiro: F. Alves.

CARDOSO de OLIVEIRA, Roberto. 1996 [1964]. O índio e o mundo dos brancos. São Paulo: Editora da UNICAMP.

CARDOSO de OLIVEIRA, Roberto. 1968. Urbanização e tribalismo: a integração dos índios Terena numa sociedade de classes. Rio de Janeiro: J. Zahar.

CARDOSO de OLIVEIRA, Roberto. 1978 [1972]. A sociologia do Brasil Indígena. Rio de Janeiro: Tempo Universitário.

CARDOSO de OLIVEIRA, Roberto. 1978. Identidade, etnia e estrutura social. São Paulo: Pioneira.

CARDOSO de OLIVEIRA, Roberto. 1979. Marcel Mauss: antropologia. São Paulo: Ática.

CARDOSO de OLIVEIRA, Roberto. 1983. Enigmas e soluções: exercícios de etnologia e de crítica. Rio de Janeiro/ Fortaleza: Tempo Brasileiro / UFC.

CARDOSO de OLIVEIRA, Roberto (org.) 1985. W. H. R. Rivers. São Paulo: Editora Ática.

CARDOSO de OLIVEIRA, Roberto. 1988. Sobre o pensamento antropológico. Rio de Janeiro/ Brasília: Tempo Brasileiro/CNPq.

CARDOSO de OLIVEIRA, Roberto. 1991. A antropologia de Rivers. Campinas, SP: Ed. da UNICAMP.

CARDOSO de OLIVEIRA, Roberto. 2002 [1991]. Razão e afetividade: o pensamento de Lucien Lévy-Brühl. 2. ed. rev. Brasília: Editora da UnB.

CARDOSO de OLIVEIRA, Roberto \& RUBEN, G. R. (orgs.). 1995. Estilos de antropologia. Campinas: Editora da Unicamp.

CARDOSO de OLIVEIRA, Roberto \& CARDOSO de OLIVEIRA, Luís Roberto. 1996. Ensaios Antropológicos sobre Moral éEtica. Rio de Janeiro: Biblioteca Tempo Universitário.

CARDOSO de OLIVEIRA, Roberto. 1998. O trabalho do antropólogo. São Paulo/ Brasília: Editora Unesp / Paralelo 15.

CARDOSO de OLIVEIRA, Roberto. 2002. Os diários e suas margens. Viagem aos territórios Terêna e Tükúna. Brasília: Editora UnB.

CARDOSO de OLIVEIRA, Roberto. 2006. Caminhos da identidade: ensaios sobre etnicidade e multiculturalismo. São Paulo: Ed. UNESP.

DILTHEY, Wilheim. 1974. Teoría de las concepciones del mundo. Madrid: Revista de Occidente. DURKHEIM, Emile. 1989. As formas elementares da vida religiosa. São Paulo: Ed. Paulinas.

FERNANDES, Florestan. 1972. O negro no mundo dos brancos. São Paulo: Difusão Europeia do Livro.

GADAMER, Hans-Georg. 1992. Verdad y Método II. Salamanca: Ediciones Sígueme.

GADAMER, Hans-Georg. 1993. Verdad y Método. Salamanca: Ediciones Sígueme. 
GALVÃO, Eduardo; WAGLEY, C. 1979. Encontro de sociedades: índios e brancos no Brasil. Rio de Janeiro: Paz e Terra.

GEERTZ, Clifford. 1989. El antropólogo como autor. Barcelona: Paidós.

HABERMAS, Jürgen. 1984. Mudança estrutural da esfera pública. Rio de Janeiro: Editora Tempo Brasileiro.

HABERMAS, Jürgen. 1989. Consciência moral e agir comunicativo. Rio de Janeiro: Biblioteca Tempo

Universitário 84, Edições Tempo Brasileiro Ltda.

KUHN, Thomas S. 1975. A estrutura das revoluções científicas. São Paulo: Perspectiva.

MAUSS, Marcel. 2003 [1950]. Sociologia e Antropologia. São Paulo: Cosac \& Naify.

RIBEIRO, Darcy. 1979. Os índios e a civilização. Petrópolis: Ed. Vozes.

Gabriel O. Alvarez

Profesor do Programa de Posgrado en Antropología Social,

Profesor Asociado da Facultad de Ciencias Sociales, Univ. Federal de Goiás (PPGAS/FCS/UFG)

Profesor visitante de la Benemérita Universidad Autónoma de Puebla, México (BUAP)

Cátedra Roberto Cardoso de Oliveira - Centro de Investigaciones y Estudios en Antropología Social / Universidad de Campinas (CIESAS/UNICAMP)

Goiania, GO - Brasil

https://orcid.org/000o-0003-3216-9428

Email: gabriel_alvarez@ufg.br 